\title{
Propuesta de un sistema de Control del Almacén
}

\section{Proposal for a Warehouse Control system}

\section{Proposta de sistema de controle de armazém}

Carlos Alberto Vásquez Villanueva carlosv@upeu.edu.pe

iD

https://orcid.org/0000-0002-0782-7364

Universidad Peruana Unión, Perú
Consuelo Sumire Bustamante

consuelosumire@upeu.edu.pe

iD https://orcid.org/0000-0002-1464-2077

Universidad Peruana Unión, Perú

\section{Rafael Romero Carazas \\ rafael.romero@upeu.edu.pe \\ https://orcid.org/0000-0001-8909-7782 \\ Universidad Peruana Unión, Perú}

Recibido: 18 de junio de 2021

Aceptado: 30 de diciembre de 2021

\section{Resumen}

El presente artículo tiene la finalidad de proponer un sistema de control de almacén, para una empresa comercial, utilizando el modelo Supply Chain Operations Reference (SCOR), organizado en cinco procesos: evaluados, diagnosticados y propuestos en diagramas de Flujo. Presenta un diseño de investigación no experimental, un nivel descriptivo-propositivo, buscando alcanzar alternativas de solución a determinados problemas y evaluar la propuesta de solución. Se realizó el diagnóstico con los lineamientos del modelo escogido; crean y aplican los procesos de acuerdo con sus experiencias, en respuesta a las necesidades de la empresa y las características del modelo: alinear, integrar, colaborar y sincronizar. Se concluye que la empresa comercializadora no posee un sistema de control, cuya propuesta ha generado la satisfacción del cliente y ventaja competitiva sobre otras empresas del mismo rubro, mejorando su rentabilidad. 
Palabras clave: Propuesta de Control; Control de Almacén; Modelo Supply Chain Operations Reference.

\begin{abstract}
The purpose of this article is to propose a warehouse control system for a commercial company, using the Supply Chain Operations Reference (SCOR) model, organized in five processes: evaluated, diagnosed and proposed in Flow diagrams. It presents a nonexperimental research design, a descriptive-purposeful level, seeking to reach alternative solutions to certain problems and evaluate the proposed solution. The diagnosis was made with the guidelines of the chosen model; They create and apply the processes according to their experiences, in response to the needs of the company and the characteristics of the model: align, integrate, collaborate and synchronize. It is concluded that the trading company does not have a control system, whose proposal has generated customer satisfaction and competitive advantage over other companies in the same field, improving its profitability.
\end{abstract}

Keywords: Control Proposal; Stock control; Supply Chain Operations Reference Model.

\title{
Resumo
}

O objetivo deste artigo é propor um sistema de controle de warehouse para uma empresa comercial, utilizando o modelo Supply Chain Operations Reference (SCOR), organizado em cinco processos: avaliados, diagnosticados e propostos em fluxogramas. Apresenta um desenho de pesquisa não experimental, de nível descritivo-proposital, buscando chegar a soluções alternativas para determinados problemas e avaliar a solução proposta. O diagnóstico foi feito com as orientações do modelo escolhido; Eles criam e aplicam os processos de acordo com suas experiências, em resposta às necessidades da empresa e às características do modelo: alinhar, integrar, colaborar e sincronizar. Conclui-se que a trading company não possui um sistema de controle, cuja proposta gerou satisfação do cliente e vantagem competitiva em relação a outras empresas do mesmo ramo, melhorando sua rentabilidade.

Palavras-chave: Proposta de Controle; Controle de estoque; Modelo de referência de operações da cadeia de suprimentos. 


\section{Introducción}

El éxito de toda organización depende cada vez más de que sus procesos empresariales se encuentren alineados con su rumbo estratégico y permitan la gestión del cambio proactivamente, la mayoría de las organizaciones carece de herramientas prácticas que permitan la identificación y gestión de sus procesos logísticos (Díaz y Delgado, 2014). Según Castillo (2019), al no contar con un sistema de control de almacén evidencia falencias en esta área; afirma que dichas falencias se ven reflejados en el control y almacenamiento de mercancías, por la falta de un buen modelo de control de almacén.

En el mismo contexto, la empresa comercial Llantas y Servicios SAC, dedicada a la compra y venta de llantas, cuyo almacén es una de las áreas consideradas el eje principal de la empresa; actualmente carece de un sistema de control de almacén, por esta razón se lleva un manejo desordenado de compras, por no realizar un procedimiento determinado, perjudicando el área de ventas, obteniendo stock con baja rotación, generando una baja rentabilidad. Otro de los factores de riesgo es la desorganización del ingreso de mercadería, al no contar con el control y procedimiento adecuado, así se ocasionaría acciones de hurto, daño o deterioro. Así mismo, el proceso de almacenaje se realiza de manera empírica, sin contar con los procesos adecuados, bajo el mismo contexto tenemos el proceso de valuación de inventarios; este es muy importante. Fuertes (2015) determina que la valuación de los inventarios en el estado de situación financiera afecta el análisis del rendimiento financiero y estas operaciones deben ser analizadas para representar fiablemente la posición financiera.

Por lo expuesto con anterioridad, esta investigación pretende el objetivo de proponer un sistema de control de almacén para la empresa Llantas y Servicios SAC, distrito de Wánchaq, 2020.

Este mismo estudio se realizó con anterioridad en los siguientes trabajos de investigación. Toro (2020) sostiene que aplicar un sistema de clasificación de inventarios $\mathrm{ABC}$, permite determinar el grado de importancia que requieren cada uno de los productos, con la finalidad de alcanzar óptimos niveles de control de los inventarios. En ese mismo contexto, Taboada et al.(2016) afirman que para optimizar el sistema de administración de inventarios se debe realizar una clasificación ABC. Al respecto, 
Fuertes (2015) precisa que la valoración de los inventarios es relevante para la presentación de la información financiera.

En función al preámbulo anterior, Carrao et al. (2020) afirman que los estudios y literatura internacional demostraron que uno de los procesos que ayudarían a construir ventajas competitivas duraderas es el Modelo de referencia en las operaciones de la cadena de suministro (SCOR).

En ese mismo orden de ideas, Rojas et al. (2016) afirman que para lograr una mejora en la gestión de inventarios, se debe realizar el método de valuación promedio ponderado, de esta manera se mejora la rentabilidad. Por su parte, Coronado (2018) afirma que un control de inventario, según Directiva $\mathrm{N}^{\circ} 002-2000$-SUNAT, debe estar basado en el método promedio ponderado, lo cual genera un mejor desempeño y mejorando su rentabilidad.

Al respecto, Santos (2019) complementa que el propósito principal y la importancia de aplicar la NIC N 2 en el procediendo de medición de los inventarios y valoración.

Por esta razón al realizar la búsqueda de modelos que se puedan utilizar para una empresa comercial, se llegó a la conclusión de que el modelo SCOR podría ser utilizado, ya es versátil para todo tipo de empresas; según Díaz y Delgado (2014), es una herramienta para representar, analizar y configurar la cadena de suministro. Proporciona un marco único que integra conceptos de negocio, indicadores de gestión y la identificación de mejores prácticas y mejorar la eficiencia en la gestión, permite describir las actividades de negocio necesarias para satisfacer la demanda de un cliente, está enfocado alrededor de los cinco procesos principales de gestión: Planificación, Aprovisionamiento, Manufactura, Distribución y Devolución o Retorno.

Por eso el proceso de compras (Planificación - Plan) que, según Organización Internacional del Trabajo (2016), dice que el éxito de sus ventas dependerá del éxito en sus compras. Si no hay planificación en las compras empresariales adecuadamente, se gastará más dinero de lo previsto, es importante llevar un control adecuado de sus existencias, para proteger su inversión, una buena planificación de compras ayuda a controlar y manejar adecuadamente el capital de la empresa y mantener un inventario razonable, para la producción sin excesos o pocas cantidades en proporción a las ventas. Seguidamente tenemos el proceso de aprovisionamiento (Source) - Ingreso de Mercadería que Iglesias (2012), define que dentro de la secuencia de actividades y una vez 
descargadas las mercancías del vehículo que las ha transportado, es preciso proceder a la comprobación de su estado, de las cantidades recibidas y a la clasificación de los productos, antes de situarlos en sus lugares de almacenamiento. Así mismo, Justino y Vargas (2018) precisan que se trata de un proceso estándar, que se aplica a cualquier tipo de entrada de mercancías. Esta función coteja los datos de la orden de compra y guía de remisión, haciendo usos de procedimientos administrativos.

Siguiendo el modelo se tiene el proceso de almacenaje (Producción - Make); para una empresa comercial, este procedimiento es muy importante, porque su principal movimiento radica en el área de almacén. Flamarique (2018) afirma que es una de las áreas más importantes de una empresa, es la zona de almacenaje, donde se guarda y se acumula la mercancía. Así mismo, en este proceso también se realiza el control de inventarios que, según Toro (2020), los inventarios son todos aquellos productos que mantiene una empresa, para la venta o también en materia prima para la producción, dependiendo de la actividad económica que realice la organización; los inventarios desempeñan un papel de gran importancia, para el desarrollo normal de las operaciones del negocio. Rojas et al. (2016) realzan la importancia del control de inventarios, para desarrollar pronósticos de ventas, de compras y/o presupuestos; también es necesario para determinar costos reales de las existencias, su recepción y almacenaje, y contabilidad.

La importancia del control de inventarios de las empresas comerciales radica precisamente en el eje de éstas: la compra y venta de bienes o servicios; un sistema de inventario inadecuado puede traer consecuencias lamentables en las utilidades de la empresa. Por otro lado, un buen manejo permitirá a la empresa saber de manera confiable cantidades y niveles, cuáles productos tienen mayor rotación, cuándo reabastecer, situación económica en cada periodo, el valor de la mercancía, para eso se mencionan algunos métodos de control: (a)Método de Control ABC. (b) Modelo Básico de Cantidad Económica de Pedido (CEP), (c) Existencias de Reservas o Seguridad de Inventarios, (d) Método Just in Time, (e) Métodos de Valuación de Inventarios; PEPS (Primeras entradas, primeras salidas) y Promedio Ponderado o Móvil. Seguidamente contamos con el proceso de salida de almacén (Distribución - Deliver) que, según Rios y Meneses (2017), el proceso de salida de los materiales por venta de los mismo, deben tener un registro indicando la fecha, la descripción del artículo vendido y el precio. Así mismo, Díaz y Delgado (2014) analizaron este proceso de gestión, relacionados con peticiones de 
clientes y envíos, con la gestión de almacén, con la recepción y verificación del producto de parte del cliente y finalmente, con la facturación.

De acuerdo con los trabajos revisados es importante indicar que el ingreso y la salida de mercadería son importantes y tener claro los procedimientos a seguir, para hacer la entrega correcta del bien. El siguiente proceso es devolución de mercadería (Devolución - Return) es el proceso, mediante el cual un cliente que ha comprado una mercancía previamente la devuelve a la tienda y, a cambio, recibe efectivo por devolución o en algunos casos, otro artículo (igual o diferente, pero de valor equivalente) o un crédito, para usar en la tienda en actividades asociadas con el flujo inverso o logística inversa, es decir, la retirada de

\section{Metodología}

La investigación planteada tiene un diseño no experimental, con diseños transeccionales (transversales) y longitudinales. La investigación es de corte transversal, porque recolectan datos en un solo momento, en un tiempo único (Hernández et al., 2014).

El nivel de investigación es descriptivo-propositiva, busca alcanzar alternativas de solución frente a determinados problemas de la realidad, se investiga una determinada realidad problemática, en término de crítica y evaluación, para proponer un modelo de solución basado en algún fundamento teórico-científico, dicho modelo de solución puede ser un proyecto, una tecnología, una estrategia o un plan de acción (Charaja, 2011).

La población está conformada por la empresa comercial Llantas y Servicios SAC y la muestra poblacional es el área de almacén. La investigación planteada permite aplicar las siguientes técnicas: análisis documentario y la encuesta.

Por otro lado, los instrumentos a utilizar son la lista de chequeo, en forma de cuestionario dicotómico y la prueba de recorrido para ver dónde implementar los controles.

Para realizar el procesamiento de la información y análisis de todos los datos, se utilizó el Microsoft Excel, y el análisis de los resultados mediante el programa SPSS, que permitirá elaborar los cuadros y/o figuras para su correspondiente interpretación. 


\section{Resultados y discusión}

\section{Diagnóstico de control de Almacén}

\section{Prueba de recorrido}

Como consecuencia de la aplicación de las técnicas de recolección de datos, se tiene la prueba de recorrido como resultado descriptivo:

Se inicia describiendo el origen de la empresa comercial Llantas y Servicios SAC, una empresa cusqueña familiar, que inicia sus operaciones el año 1980, fundada por su Director Víctor Málaga, se dedica a la comercialización de Neumáticos (Llantas) y Conexos (Cámaras, Protectores, Accesorios), así como a la prestación de Servicios de Mecánica Ligera relacionados con este rubro. Actualmente, su hijo Mauricio Málaga es el gerente de dicha empresa, tiene 15 trabajadores en planilla y está en régimen general.

Después de un breve resumen narrada por el gerente, se empieza la realización de la prueba de recorrido acompañada del personal de compras, iniciando con la primera dimensión que involucra directamente al área de compras; la empresa no posee procesos para realizar compras; la mayor parte estas compras son manejadas por el gerente: uno de los accionistas; el personal a cargo manifiesta que los proveedores se contactan directamente con el gerente, a fin de realizar cierre de mes exitoso para ellos, por eso las compras no siguen procedimientos de estimación de demanda, ni consultas de stock; este proceso es nulo cuando el gerente entabla la comunicación con el proveedor y los pedidos son realizados de manera desmedida, para complacer la solicitud de los proveedores y, en muchos casos, se compra mercadería de baja rotación; así mismo, no se cuenta con ningún plan de compras mensual, el personal de ventas se encuentra disconforme con este proceso.

De la misma manera, realizamos una prueba de recorrido del proceso de ingreso de mercadería; el personal encargado de compras y almacén manifestaron que a veces se pide en exceso, porque el proveedor realiza campañas, descuentos y, al contactar directamente con la gerencia, se realizan compras de gran volumen, sin medir la capacidad de sus almacenes; esto dificulta, en gran manera, el ingreso, también manifestaron que cuentan con un procedimiento empírico de ingreso de mercadería que tratan de cumplir; pero no tienen ningún procedimiento oficial, cuando realizamos la prueba de recorrido coincidimos en un ingreso de mercadería, observando que efectivamente se realiza la verificación de las especificación técnicas solicitadas en la 
orden de compra (Marca, modelo, medida, aplicación); sin embargo, al realizar cuestionamientos de este tema indican que cuando se compra en gran cantidad no se llega a realizar esta buena práctica; sin embargo, sí se llegan a verificar al menos la marca, medida y la cantidad de mercadería recibida; de igual manera se cuestionó la ubicación del almacén, para la recepción de productos; manifiesta el almacenero que el lugar correcto para realizar la recepción de mercadería debería ser el almacén - II (Av. Huayruropata); este cuenta con la infraestructura adecuada; sin embargo, el ingreso del transportista es complicado, se encuentra en una Av. de alta circulación vehicular; por eso las descargas deben realizarse muy temprano para evitar el malestar del almacenero.

Se realizó la prueba de recorrido para el proceso de almacenaje junto con el personal encargado del área de almacén, quien afirmó no tener un proceso adecuado de ingreso de mercadería; desconoce de procedimientos de almacenaje; también consultamos acerca de la toma de inventario y precisó que la toma de inventario se realiza anualmente al igual que el registro de pérdidas y desmedros; esta toma es realizada para el balance anual y alcanzada al contador. Así mismo, consultamos acerca de la valuación de inventarios; él respondió que no se tiene el personal encargado, para realizar este tipo de procesos diariamente y que la empresa cuenta con contabilidad externa, siendo esta área la encargada de realizar esto anualmente.

En ese mismo contexto se realizó la prueba de recorrido al proceso de salida de almacén, junto con el personal encargado del área de almacén y el personal de ventas; estos manifiestan que se tiene un procedimiento de salida de almacén empírico, creado bajo sus propias experiencias, existiendo varias falencias en este proceso, por eso se debe contar con un proceso de salida de almacén.

También se consultó si se cuenta con registro de salida de almacén; manifestaron que sí se realiza un registro en Microsoft Excel y solo lo maneja el área de almacén. Acerca de la infraestructura manifestaron contar con esta; sin embargo, no cumple con estándares de seguridad y orden, afirmando que no solo el almacenero ingresa a sacar productos, sino cualquier personal de la empresa. Así mismo, se les cuestionó acerca de la ubicación de los dos almacenes, manifestaron que la ubicación de ambos es cercana; sin embargo, la distribución de mercadería en estos dos almacenes es un problema; este procedimiento no ayuda a atender eficazmente a los clientes. 
Del mismo modo, se consultó si la cantidad de almacenes cumple con la demanda de clientes, declararon que realmente no es la cantidad de almacenes la que no responde a la demanda, sino el inadecuado proceso de compras que se sigue.

Por último, se realizó la prueba de recorrido al proceso de devolución de mercadería, conjuntamente con el almacenero, quien revela que realmente no existe un procedimiento de devolución de mercadería, suele suceder que el cliente devuelve una mercadería y no se hace envió al proveedor generando una molestia al cliente.

\section{Diagnóstico del control de almacén por medio de la encuesta}

Como consecuencia de la aplicación de las técnicas de recolección de datos, la encuesta fue realizada a un total de 15 personas y con un total de 30 preguntas, de ese total de preguntas se seleccionaron 5 logrando identificar y analizar lo siguiente:

\begin{tabular}{|c|c|c|c|c|}
\hline \multirow{3}{*}{ Ítems } & \multicolumn{4}{|c|}{ Resultados } \\
\hline & \multicolumn{2}{|c|}{ Absolutos } & \multicolumn{2}{|c|}{ Relativos } \\
\hline & NO & SI & NO & SI \\
\hline $\begin{array}{l}\text { ¿La empresa cuenta con un procedimiento } \\
\text { de estimación de la demanda para la } \\
\text { realización de las compras? }\end{array}$ & 13 & 2 & $86,7 \%$ & $13,3 \%$ \\
\hline $\begin{array}{l}\text { ¿La empresa cuenta con procedimientos } \\
\text { para realizar la logística de ingreso? }\end{array}$ & 11 & 4 & $73,3 \%$ & $26,7 \%$ \\
\hline $\begin{array}{l}\text { ¿La empresa cuenta con procedimientos } \\
\text { para realizar la valuación de inventario? }\end{array}$ & 13 & 2 & $86,7 \%$ & $13,3 \%$ \\
\hline $\begin{array}{l}\text { ¿La logística interna de la empresa cuenta } \\
\text { con procedimientos para el almacenamiento } \\
\text { de la mercadería? }\end{array}$ & 11 & 4 & $73,3 \%$ & $26,7 \%$ \\
\hline $\begin{array}{l}\text { ¿La empresa cuenta con procedimientos de } \\
\text { gestión de salida? }\end{array}$ & 8 & 7 & $53,3 \%$ & $46,7 \%$ \\
\hline $\begin{array}{l}\text { ¿La empresa cuenta con procedimientos } \\
\text { para la devolución de mercadería? }\end{array}$ & 13 & 2 & $86,7 \%$ & $13,3 \%$ \\
\hline
\end{tabular}




\section{Propuesta de control de almacén}

Durante la evaluación descriptiva y estadística, se llegó a discernir que la empresa requiere un sistema de control de almacén, por eso se plantea la siguiente propuesta de un sistema de control de almacén, siguiendo los lineamientos del modelo Supply Chain Operations Reference model - SCOR; este modelo fue desarrollado en Estados Unidos en el año de 1996 por Supply Chain Council, a partir de la iniciativa de 69 empresas que voluntariamente acuerdan desarrollar un estándar aplicable a los procesos operativos de negocio, y permitiera obtener eficiencia y productividad. En la actualidad, esta asociación está compuesta por más de 1000 socios, empresas de todo tipo de sectores, empresas fabricantes, distribuidores, transportistas, desarrolladores, empresas de consultoría, asociaciones gubernamentales, etc.

Los beneficios que hemos analizado y que podría favorecer a la empresa comercial donde se plantea la propuesta son:

$\checkmark$ Optimizar y reducir costos.

$\checkmark \quad$ Implantar la gestión de procesos, integrando distintas áreas de la empresa.

$\checkmark \quad$ Mejorar la calidad, eficiencia y el nivel de servicio ofrecido.

$\checkmark$ Permite retroalimentar, corregir y redefinir los procesos.

$\checkmark$ Proporciona un lenguaje común a adaptable a la empresa, abarcando todos los procesos claves: Compras (Planificación/Aprovisionamiento), Almacenaje (), Salida de almacén (), Devolución o reclamo de productos ().

$\checkmark \quad$ Facilidad de uso y rapidez de implementación.

$\checkmark \quad$ Flexibilidad ya que se adapta perfectamente a cualquier sector y complejidad de la cadena.

$\checkmark \quad$ Este modelo no solo propone un modelo de referencia de evaluación de la cadena de suministros optima, si no también hace sugerencia sobre herramientas de control que se pueden aplicar en los diferentes procesos: A continuación, presentare los flujogramas y la descripción de cada uno de estos procesos: 
Figura 1

Procesos según modelo Supply Chain Operations Reference model SCOR

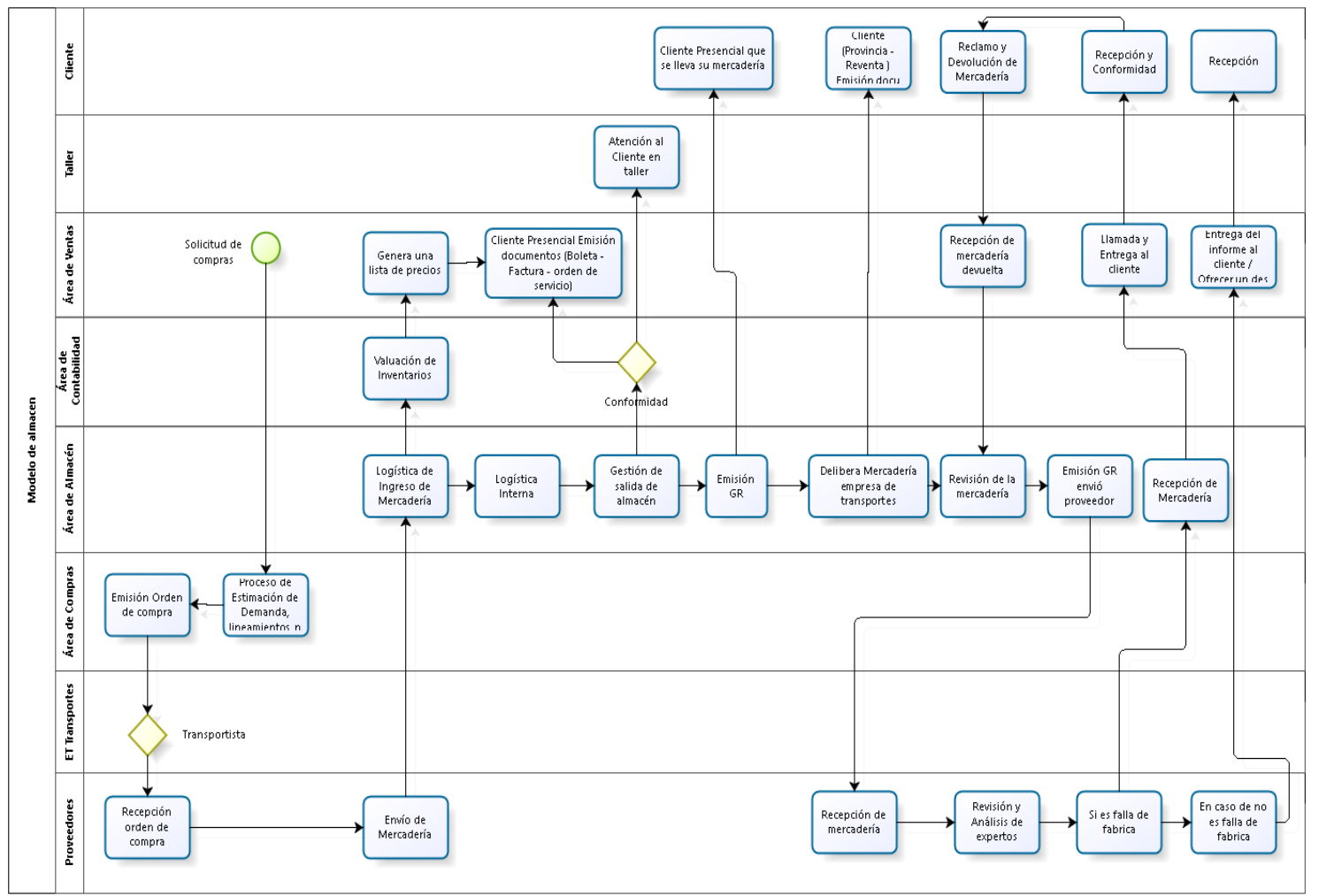

Este flujograma engloba los cinco procesos planteados del sistema de control de almacén. Bajo este flujograma se desea alinear, integrar, colaborar y sincronizar los procesos. El modelo SCOR es una metodología, para conocer, evaluar y mejorar los procesos de planeación y ejecución de una cadena de suministros, desde el proveedor del proveedor hasta el cliente del cliente. 


\section{Figura 2}

\section{Proceso de Compras}

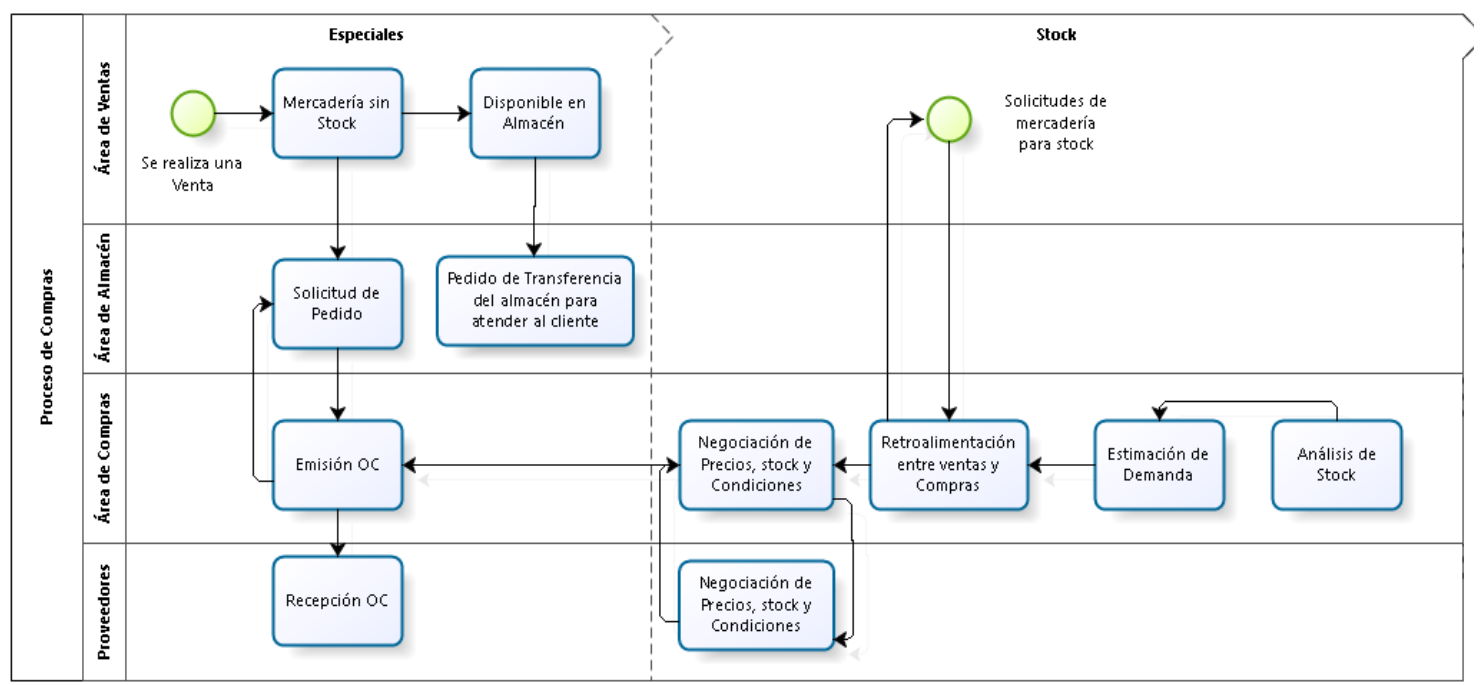

$\checkmark \quad$ El área de ventas solicita mercadería a almacén.

$\checkmark$ Se determina qué tipo de compra es 1) Pedido especial para un cliente determinado, 2) Pedido para stock se recomienda que este último sea de manera quincenal para el correcto abastecimiento.

$\checkmark$ Seguidamente esta compra deberá ser planeado en este proceso debe estar inmerso la estimación de la demanda, verificación de los lineamientos entre demanda y abastecimiento, verificación de stock, estos tres subprocesos tienen como objetivo medir el grado de planificación de la demanda, el uso de herramientas idóneas y la disponibilidad de la información, esto por parte del área de almacén en coordinación con el área de compras

$\checkmark \quad$ El área de compras debe mantener comunicación con el proveedor y ver la negoción de precio, stock y condiciones, para este proceso después de realizar la verificación documentaria se diseñó una base de datos de los proveedores.

$\checkmark \quad$ Envió de Orden de Compra vía correo.

\section{Nota:}

Las ventajas con las que se puede contar en este proceso son mayor eficacia en las negociaciones, mayor control en la gestión con proveedores, reducción de costos, mejora la gestión de inventarios. 


\section{Figura 5}

\section{Proceso de Ingreso de Mercadería}

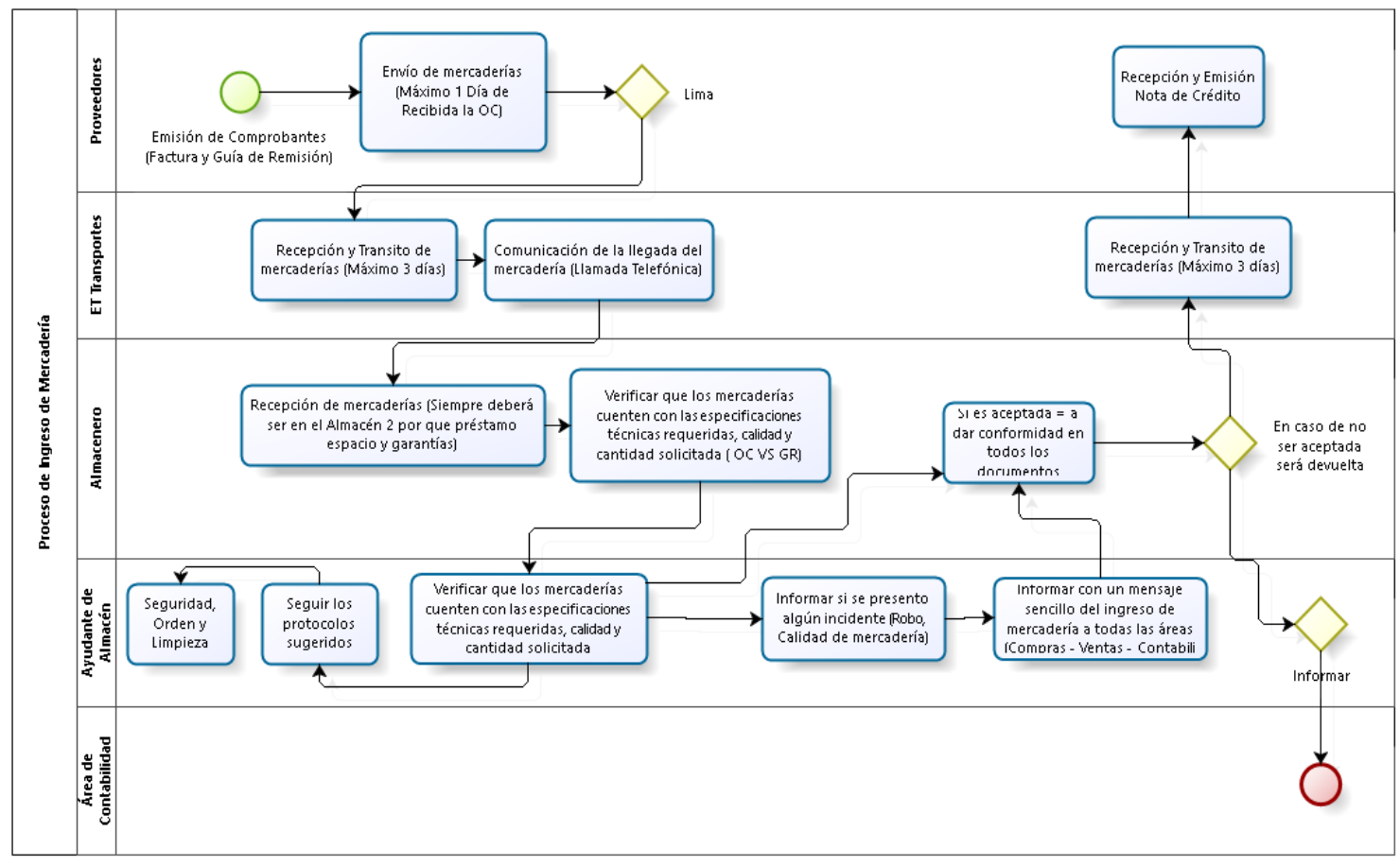

El proceso de ingreso de mercadería, se da inicio con la conformidad de la recepción de la Orden de Compra de parte del proveedor; este mismo emitirá factura y guía de remisión, para concluir con el despacho de la mercadería, para efectos contables esta será considera en tránsito.

$\checkmark$ Después de 3 días, el transportista se comunicará con el almacén II para realizar la entrega de la mercadería, este almacén, según el diagnóstico, es considerado el ideal, porque presta las condiciones de infraestructura y de personal necesarias, a fin de evitar hurtos, pérdidas al momento de realizar el ingreso de mercadería,

$\checkmark$ Recepción de mercadería el almacenero realizará la verificación de las especificaciones solicitadas en la orden de compra, de no ser el caso este será comunicado al área de compras y devuelto inmediatamente al proveedor.

$\checkmark$ En caso de que todo sea conforme se procederá a la recepción y conformidad, seguidamente se comunicará a todas las áreas ventas, compras y contabilidad, para que esta información sea más rápida y fluida será realizada mediante un grupo de WhatsApp, donde se enviará una foto de la guía de remisión y el detalle de la cantidad Anexo. 


\section{Nota:}

$\checkmark$ En caso de que suceda algún incidente de hurto, pérdida o mercadería dañada deberá ser registrada y comunicada al área de contabilidad.

\section{Figura 7}

\section{Proceso de almacenaje}

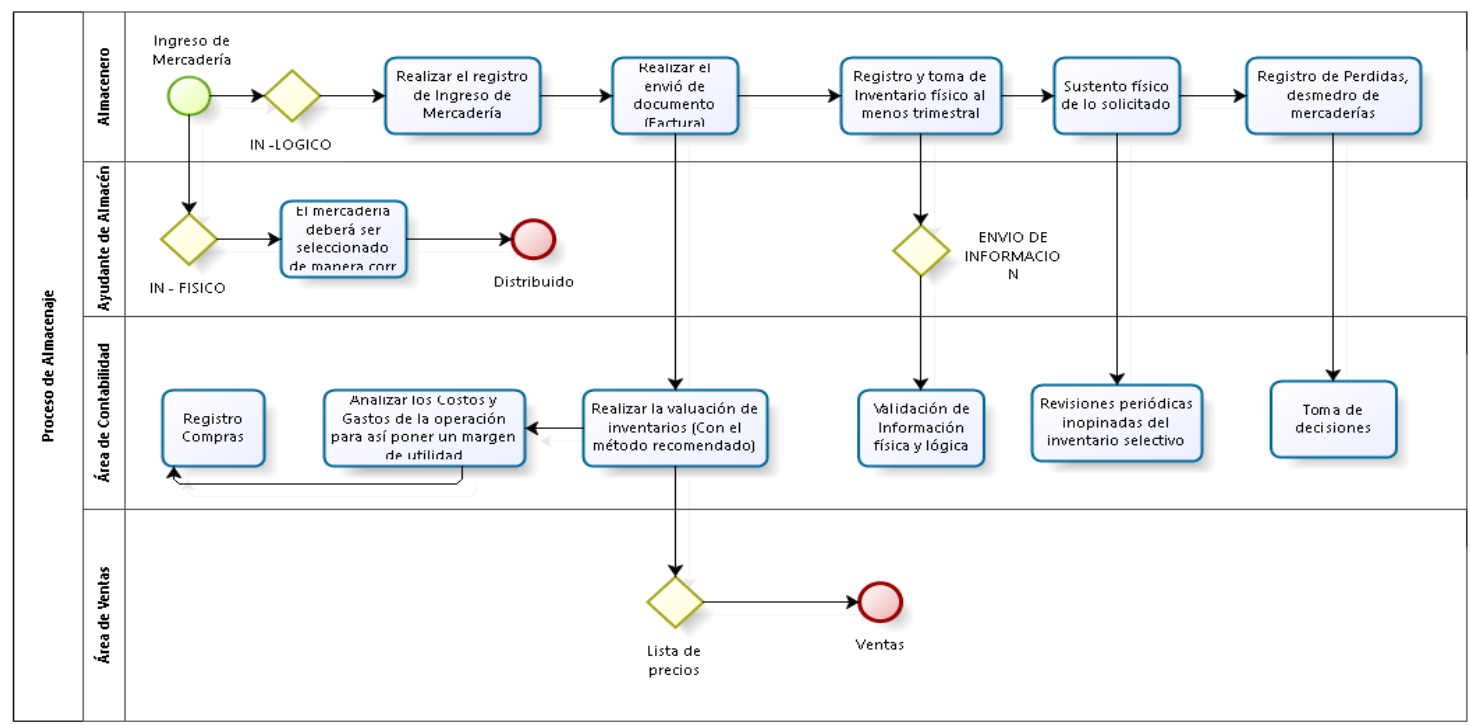

$\checkmark$ El proceso de almacenaje consiste en realizar el ingreso de la mercadería en físico y lógico.

$\checkmark$ Ingreso físico de la mercadería deberá ser seleccionada y distribuida, de acuerdo con el modelo sugerido que es el $\mathrm{ABC}$ anexo.

$\checkmark$ Paralelamente se realizará el ingreso lógico iniciando con el registro de mercadería que será realizado por almacén.

$\checkmark$ Se realizará él envió de los documentos guía de remisión y facturas al área de contabilidad.

$\checkmark$ El área de contabilidad realizará la valuación de existencias bajo el método promedio ponderado.

$\checkmark$ El área de contabilidad analizará costos y gastos, para determinar el margen de utilidad y así generar una lista de precios para el área de ventas. 


\section{Nota:}

$\checkmark$ De manera inopinada el área de contabilidad realizara revisiones periódicas de stock al área de almacén.

$\checkmark \quad$ El área de almacén debe realizar un registro de desmedros, perdidas y este mismo debe de ser informado al área de contabilidad en la fecha de la ocurrencia.

\section{Figura 8}

\section{Proceso de salida de almacén}

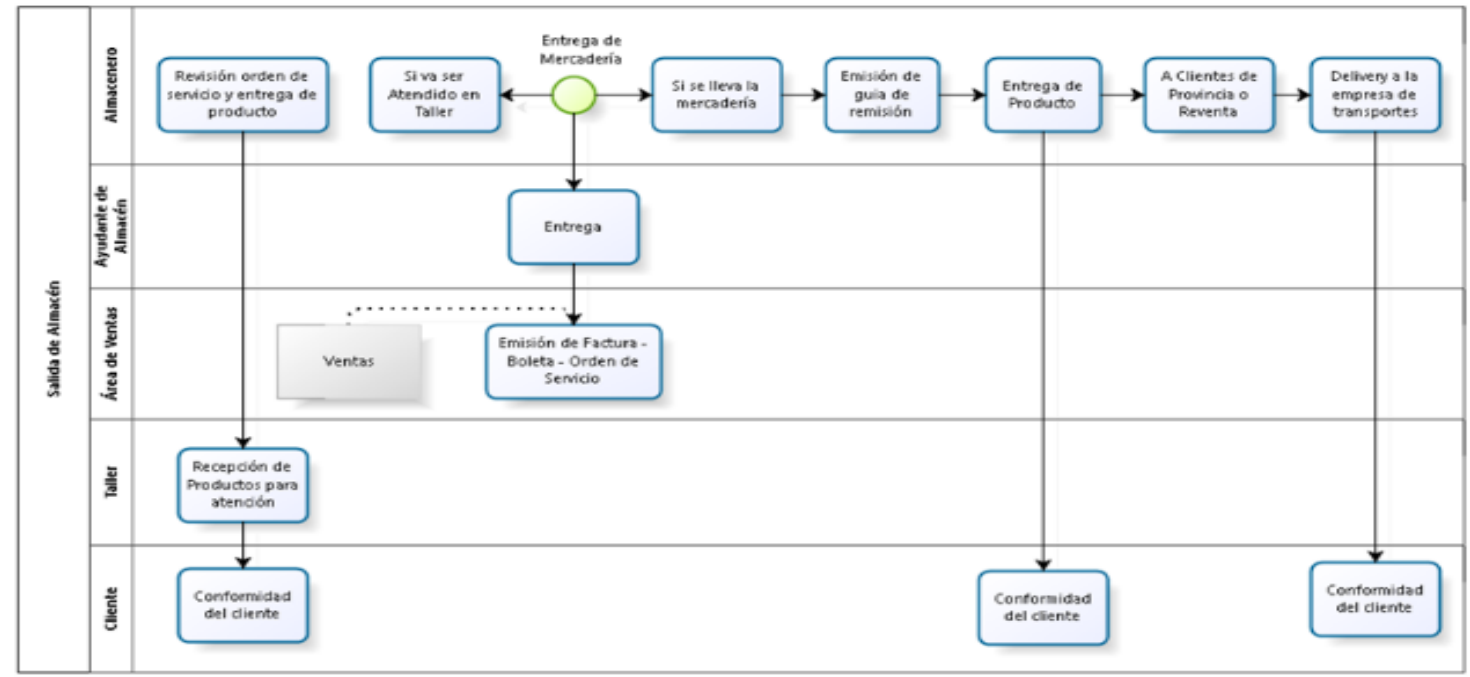

Figura 8: Proceso de salida de almacén

$\checkmark$ El proceso de salida de almacén se iniciará por el área de ventas, generando documentos (Orden de Servicio, factura o boleta); este mismo tiene tres tipos de salida:

$\checkmark$ Cliente que compra el producto en taller, la entrega de la mercadería será realizada con una orden de servicio al jefe de taller; este mismo deberá recibir la conformidad del cliente y seguido se realizará la emisión del comprobante.

$\checkmark$ Cliente que compra el producto en tienda, será entregado por almacén con la factura o boleta seguidamente se emitirá una guía de remisión para el traslado de la mercadería.

$\checkmark$ Cliente de provincia o reventa al recibir la factura o boleta emitida por el área de ventas.

$\checkmark$ Este tercer tipo de salida, el almacenero deberá realizar las coordinaciones necesarias, para el envío de la mercadería por la empresa de transportes determinada por el cliente; así mismo, el área de almacén deberá utilizar el equipo de transporte, para 
realizar el envío de esta mercadería, debe existir una buena comunicación con el cliente para poder así cumplir con sus expectativas y recibir su conformidad.

\section{Figura 9}

Proceso de devolución de mercadería

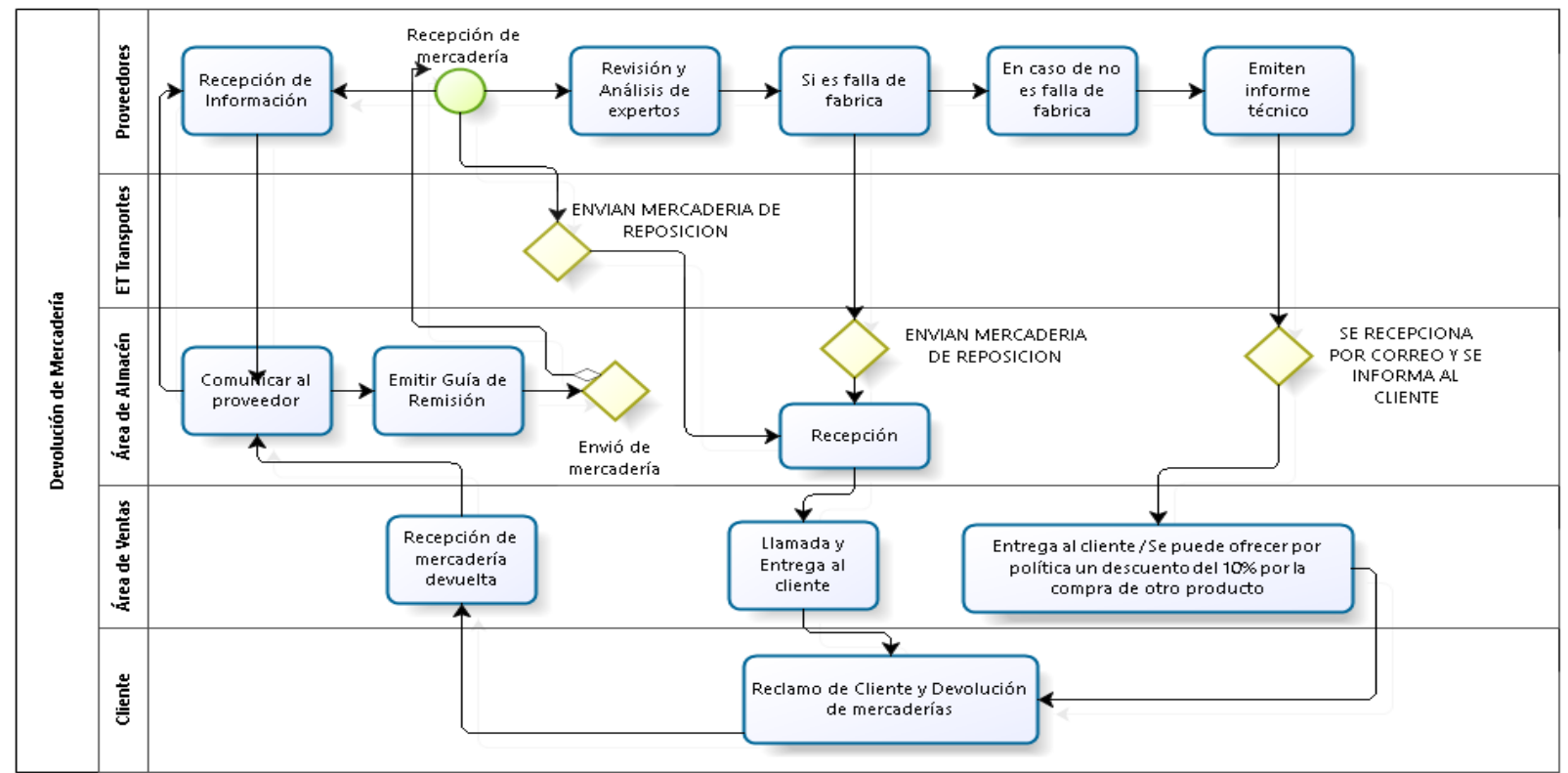

$\checkmark$ El proceso de devolución de mercadería, inicia con el reclamo de cliente y devolución de mercadería al área de ventas, que esta a su vez informa al área de almacén.

$\checkmark \quad$ El área de almacén deberá revisar y se emiten un pre informe.

$\checkmark$ El área de almacén debe informar de este suceso al área de compras.

$\checkmark$ El área de compras se pondrá en contacto con el proveedor sobre la mercadería.

$\checkmark$ El área de almacén en coordinación deberá emitir una guía de remisión y realizar la devuelta a las instalaciones del proveedor.

$\checkmark$ El área de compras debe realizar seguimiento a este proceso, para obtener la emisión de un informe de expertos que podría tener dos resultados.

$\checkmark$ El primero determina que es falla de fábrica y la mercadería es reemplazada, enviada y recepcionada por el área de almacén, que a su vez informa al área de 
ventas, para que se comunique con el cliente y coordinar el remplazo de la mercadería.

$\checkmark$ La segunda probabilidad es que sea denegada la solicitud del cliente y el proveedor envié el informe sobre la negativa al área de ventas; esta se debe comunicar al cliente y exponer de manera clara la negativa de su solicitud; para este tipo de situaciones sugerimos que se haga un descuento del $10 \%$ por la compra de la mercadería a reponer, para mantener una buena relación con el cliente.

\section{Conclusiones}

Después de analizar el proceso de compras, se evidenció que la empresa no cuenta con este proceso; se necesita elaborar un plan que permita estimar la demanda futura, con la finalidad de alinear el abastecimiento con la demanda, reduciendo el inventario inmovilizado y la pérdida de ventas por falta de stock.

De la misma manera, se analizó el proceso de ingreso de mercadería; se evidenció la necesidad de poseer un procedimiento de ingreso y con políticas respectivas. Así mismo, no se tiene un registro adecuado de ingreso de mercadería, evidenciando la falta de data.

La empresa actualmente no dispone de un sistema contable, el mismo que permita conocer los productos en stock, saber qué productos se necesitan en almacén y atender las necesidades del cliente en el momento cuando se realice la compra.

Después de analizar el proceso de almacenaje, se evidenció la ausencia de este; por lo tanto, se plantea un proceso siguiendo el modelo ABC.

En el almacén no se ejecuta inventarios físicos de la mercadería de manera constante, a pesar de que los mismos juegan un papel muy importante en la empresa y necesitan que este procedimiento sea perfeccionado

Después de analizar el proceso de salida de almacén, se evidencio la falta de este proceso. Después de analizar el proceso de devolución o reclamo de productos se evidencio la falta de este. En este sentido, se plantea la elaboración de un sistema de control de almacén; se mostró que el modelo de referencia sugerido Supply Chain Operations Reference, model - SCOR puede ser aplicado en cada uno de los procesos y 
subprocesos del modelo de control de almacén. Los procesos planteados son de fácil implementación y acordes a la realidad operacional de la empresa.

Mediante la propuesta del control de almacén, con la ayuda del modelo Supply Chain Operations Reference, model - SCOR, se identificó los procesos que se deben de mejorar, principalmente el proceso de planificación de compras. ya que se observó gran cantidad de mercadería de baja rotación.

El diseño de un Sistema de control de control de almacén de la empresa Llantas y Servicios SAC, ayudará a mejorar la gestión de inventarios, con el objetivo de disminuir costos, riesgos, y gastos innecesarios, aumentar las ventas y, por ende, aumentar las utilidades al final del periodo; esta herramienta se realizó en base a los resultados obtenidos de las respectivas encuestas efectuadas a los implicados del almacén, en el cual se puede descubrir que la inexistencia de controles adecuados les ha ocasionado problemas en las actividades que se desarrollan cotidianamente. Esta herramienta ayudará a la empresa alcanzar las expectativas planteadas como empresa y satisfacción de los requerimientos de sus clientes

La investigación realizada al Almacén de Llantas y Servicios SAC ha permitido conocer la falta de políticas, procedimientos, código de ética, que permitan al personal realizarse de la mejor manera en sus funciones y puedan crecer como empresa.

\section{Referencias bibliográficas}

Carraro, E., Oliveira, U., y Thielmann, R. (2020). Estudio y análisis para la difusión del Modelo de Referencia en Operaciones de la Cadena de Suministro (SCOR) en universidades y organizaciones. Revista Valore, Volta Redonda.

https://doi.org/10.1017/CBO9781107415324.004

Castillo, K. (2019). Propuesta de un Modelo de Optimización para mejorar la eficiencia en el procesode traslados de puntos de venta a almacenes principales de la empresa Helados Panameña SA [Universidad de Bogotá Jorge Tadeo Lozano]. https://doi.org/10.1017/CBO9781107415324.004

Charaja Cutipa, F. (2011). El MAPIC en la Metodologia de Investigación. Sagitario Impresores.

Coronado, P. (2018). Propuesta de Control de Inventarios, según Directiva $N^{\circ} 002$ - 
2000-SUNAT, en Hapcom SAC, Distrito de Tacna, 2018 [Universidad Peruana Unión].

https://repositorio.upeu.edu.pe/bitstream/handle/UPEU/1575/Patricia_Tesis_Licen ciatura 2018.pdf? sequence $=5 \&$ is Allowed $=y$

Díaz, C., y Delgado, M. (2013). El Modelo Scor y El Balanced Scorecard, Una Poderosa Combinación Intangible Para La Gestion Empresarial Scor Model and the Balanced Scorecard, a Powerful Combination for Business Management Assets. Vision de Futuro.

https://www.researchgate.net/publication/317534513_El_modelo_SCOR_y_el_Bal anced_Scorecard_una_poderosa_combinacion_intangible_para_la_gestion_empres $\underline{\text { arial }}$

Flamarique, S. (2018). Diseño y organización del almacén. In Gestión de existencias en el almacén.

https://books.google.com.pe/books/about/Gestión_de_existencias_en_el_almacén. $\underline{\mathrm{htm} l}$ ?id $=\mathrm{CDd} 8 \mathrm{DwAAQBAJ} \& \mathrm{printsec}=$ frontcover\&source $=\mathrm{kp} \_$read_button\&redir $\underline{\mathrm{esc}}=\mathrm{y} \# \mathrm{v}=$ onepage $\& \mathrm{q} \& \mathrm{f}=$ false

Fuertes, J. A. (2015). Métodos, técnicas y sistemas de valuación de inventarios. Un enfoque global. Gestión Joven. https://doi.org/1988-9011

Hernández, R., Fernández, C., y Baptista, M. del P. (2014). Metodologia de la Investigación. In MC Graw Hill Education.

https://doi.org/10.1017/CBO9781107415324.004

Iglesias, A. (2012). Manual de Gestión de Almacenes. In Manual de gestión de almacenes. https://search.proquest.com/docview/2260024353? accountid=31491

Justino, H., y Vargas, R. (2018). Propuesta de un Sistema de Gestion de Almacenes para Mejorar la Productividad en la Empresa Danper Trujillo SAC 2018. Universidad Privada Antenor Orrego.

Rios, J., y Meneses, G. (2017). Diseño de una Propuesta de Mejoramiento para la Gestion y Control de Inventarios del “Almacen Michelin.” Universidad Catolica de Pereira.

Rojas, M., Muñoz, J., y Cáceres, M. (2016). Propuesta para la mejora en la Gestión de Inventarios de la Empresa Casa Musical E.I.R.L. Arequipa, 2016 [Universidad 
Tecnológica del Perú]. http://repositorio.utp.edu.pe/handle/UTP/1122\%0A

Santos, K. (2019). Aplicación Contable de la NIC 2 Inventarios, en una empresa distribuidora tipo SAC de Arequipa 2017. Universidad Católica de Santa María.

Taboada, P., Aguilar, Q., Ibarra, J., y Ramírez, M. (2016). Optimización de un Sistema de Abastecimiento de Pintura a Consesionarios de Baja y Media Demanda. Informacion Tecnologica. https://doi.org/10.4067/S0718-07642016000300006

Toro, G. (2020). Diseño de un Sistema de Control de inventarios ABC para la empresa la empresa Vanobri Distribuciones S.C., del Cantón Santo Domingo, Provincia de Santo Domingo de los Tsachilas [Escuela Superior Politécnica de Chimborazo]. http://dspace.espoch.edu.ec/handle/123456789/14024\%0A 